\title{
GALLIUM NITRIDE ON GALLIUM OXIDE SUBSTRATE FOR INTEGRATED NONLINEAR OPTICS
}

\author{
Kashif M. Awan and Ksenia Dolgaleva \\ School of Electrical Engineering and Computer Science \\ University of Ottawa \\ Ottawa, Canada \\ Ksenia.Dolgaleva@uottawa.ca
}

\author{
Mufasila M. Muhammed and Iman S. Roqan \\ Physical Sciences and Engineering Division \\ King Abdullah University of Science and Technology \\ Thuwal, Saudi Arabia \\ iman.roqan@kaust.edu.sa
}

\begin{abstract}
Keywords - nonlinear optics; integrated photonic circuits; four-wave mixing; all-optical wavelength conversion; GaN epitaxial growth component, semiconductor fabrication, plasma etching
\end{abstract}

Gallium Nitride $(\mathrm{GaN})$, being a direct bandgap semiconductor with a wide bandgap and high thermal stability, is attractive for optoelectronic and electronic applications. Furthermore, due to its high optical nonlinearity-the characteristic of all III-V semiconductors - $\mathrm{GaN}$ is also expected to be a suitable candidate for integrated nonlinear photonic circuits for a plethora of applications, ranging from on-chip wavelength conversion to quantum computing. Although $\mathrm{GaN}$ devices are in commercial production, it still suffers from lack of a suitable substrate material to reduce structural defects like high densities of threading dislocations (TDs), stacking faults, and grain boundaries. These defects significantly deteriorate the optical quality of the epi-grown GaN layer, since they act as non-radiative recombination centers. Recent studies have shown that GaN grown on $(-201) \beta$ Gallium Oxide $\left(\mathrm{Ga}_{2} \mathrm{O}_{3}\right)$ has superior optical quality due to a better lattice matching as compared to $\mathrm{GaN}$ grown on Sapphire $\left(\mathrm{Al}_{2} \mathrm{O}_{3}\right)$ [1-3]. In this work, we report on the fabrication of $\mathrm{GaN}$ waveguides on $\mathrm{Ga}_{2} \mathrm{O}_{3}$ substrate and their optical characterization to assess their feasibility for efficient four-wave mixing (FWM).

GaN waveguides on Gallium Oxide were designed using Lumerical Mode Solutions' eigensolver. The design was optimized for a better mode confinement that would allow for enhanced nonlinear optical interactions [4]. GaN was epitaxially grown on (-201) $\beta-\mathrm{Ga}_{2} \mathrm{O}_{3}$, and commercially available high quality $\mathrm{GaN}$ on $\mathrm{Al}_{2} \mathrm{O}_{3}$ was purchased for comparative studies. Initial material characterization showed a much lower lattice mismatch of $\sim 4.7 \%$ between (201) $\beta-\mathrm{Ga}_{2} \mathrm{O}_{3}$ and the $\mathrm{GaN}$ film, compared to $\sim 14 \%$ between $\mathrm{Al}_{2} \mathrm{O}_{3}$ and $\mathrm{GaN}$ [1, 5]. Room temperature photoluminescence study further showed that Gallium Oxide substrate has better optical quality than Sapphire, as shown in Fig.1 (a).

$\mathrm{GaN}$ on (-201) $\beta-\mathrm{Ga}_{2} \mathrm{O}_{3}$ wafer was first patterned with the designed waveguides using hard mask and subsequently dry-etched using ICP-RIE system. Both patterning and etching parameters were optimized to reduce roughness on the etched surface with the potential of reducing the propagation losses due to scattering. Furthermore, the sidewall roughness was reduced by employing a postprocessing wet etching technique. Scanning Electron Microscopy (SEM) images of as-etched and post-processed $\mathrm{GaN}$ waveguides are shown in Fig.1 (b).

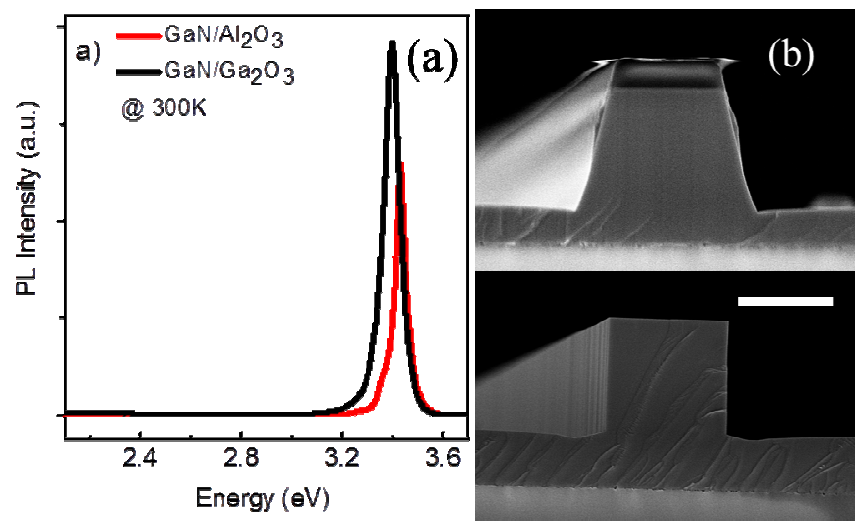

Fig. 1: (a) Room temperature photoluminescence data for GaN grown on Sapphire and Gallium Oxide. (b) SEM images of cross-section of as-etched (top) and post-processed (bottom) $\mathrm{GaN}$ waveguides, scale bar represents $1 \mu \mathrm{m}$.

The optical characterizations of the fabricated waveguides are currently in progress. The aim is to experimentally demonstrate that the improvement in the optical quality, offered by the growth of the lattice-matched $\beta-\mathrm{Ga}_{2} \mathrm{O}_{3}$ substrate, the optimization of the waveguide geometry and fabrication process can enhance FWM efficiency. This study can be a stepping stone toward realizing first on-chip source of correlated photon pairs in the visible spectral range.

\section{REFERENCES}

[1] N. V. Edwards, et al., III-Nitride Semiconductors: Electrical, Structural and Defects Properties , pp. 287-337 (2000)

[2] M. M. Muhammed, et al., Applied Physics Letters 105, 042112 (2014)

[3] K. Shimamura, et al., Japanese Journal of Applied Physics 44, L7-L8 (2005)

[4] K. Dolgaleva, et al., Optics Express 23, 22477-22493 (2015)

[5] M. M. Muhammed, et al., Scientific Reports 6 (2016) 